1 Universidade Federal do Paraná (UFPR), Programa de Pós-Graduação em Enfermagem - Curitiba (PR), Brasil. Secretaria de Estado da Saúde (Sesa), Divisão de Vigilância do Programa de Imunização Curitiba (PR), Brasil. nanda.cwk@gmail.com

2 Universidade Federal do Paraná (UFPR), Programa de Pós-Graduação em Enfermagem e Núcleo de Estudos em Saúde Coletiva (Nesc) - Curitiba (PR)

Brasil.

liliana@ufpr.br

3 Universidade Federal do Paraná (UFPR), Programa de Pós-Graduação em Enfermagem e Núcleo de Estudos em Saúde Coletiva (Nesc) - Curitiba (PR),

Brasil.

mnolascochaves@gmail.com

\section{Perdas evitáveis de imunobiológicos na instância local: reflexões acerca do processo de trabalho da enfermagem}

\author{
Avoidable losses of immunobiologicals in the local instance: \\ reflections on the nursing work process
}

Fernanda Crosewski, Liliana Müller Larocca ${ }^{2}$, Maria Marta Nolasco Chaves ${ }^{\mathbf{3}}$

RESUMO Objetivou-se identificar a ocorrência de perdas evitáveis de imunobilógicos em um município, no período de 2011 a 2015, e conhecer o processo de trabalho da equipe de enfermagem na Rede de Frio (RF). Trata-se de um estudo de caso do tipo exploratório documental e de campo, com abordagem quantitativa e qualitativa. Os dados foram extraídos dos Sistemas de Informações de Apuração de Imunobiológicos Utilizados (SI-AIU) e do Programa Nacional de Imunização (PNI) e coletados em 54 entrevistas com profissionais de enfermagem. Das ocorrências evitáveis, destaca-se a perda por validade vencida (83\%). Algumas estratégias de enfrentamento foram propostas, com destaque para gerenciamento de lotes na instância superior.

PALAVRAS-CHAVE Enfermagem. Imunização. Refrigeração.

ABSTRACT The objective of this study was to identify the occurrence of avoidable losses of immunologicals in a municipality, in the period from 2011 to 2015, and to know the work process of the nursing team in the Cold Network. It is a case study of the documental exploratory and field type, with quantitative and qualitative approach. Data were extracted from the Information Systems of Verification of Immunobiological Used (SI-AIU) and from the National Immunization Program (PNI), and collected in 54 interviews with nursing professionals. Of the avoidable occurrences, it is highlighted the loss due to expired validity (83\%). Some coping strategies were proposed, with emphasis on batch management in the higher instance.

KEYWORDS Nursing. Immunization. Refrigeration. 


\section{Introdução}

O Ministério da Saúde instituiu, em 1973, o Programa Nacional de Imunizações (PNI), cujos técnicos são responsáveis por coordenar as atividades de imunização, utilizando-o tanto para estratégias básicas de vacinação de rotina como para campanhas anuais, contribuindo, assim, para o controle, a eliminação e/ou erradicação das doenças imunopreveníveis $\mathbf{1}^{\mathbf{1 , 2}}$.

No decorrer das últimas décadas, as vacinas representaram para a saúde pública o método de proteção específica com a melhor relação custo-benefício. Em consequência, observou-se a mudança do perfil epidemiológico das doenças imunopreveníveis, com diminuição do número de casos, reduzindo, assim, custos com hospitalizações e tratamentos ${ }^{3}$.

A efetividade desse processo está diretamente ligada à Rede de Frio (RF) - processo de armazenamento, conservação, manipulação, distribuição e transporte dos imunobiológicos, onde são garantidas as condições adequadas de refrigeração, desde o laboratório produtor até sua administração. Quaisquer falhas podem representar perda dos produtos e deficiências na proteção dos indivíduos ${ }^{4,2}$.

Toda a estrutura da RF possibilita que os imunobiológicos fiquem conservados a uma temperatura entre $+2^{\circ} \mathrm{C} \mathrm{e}+8^{\circ} \mathrm{C}$ na instância local ou $-20^{\circ} \mathrm{C}$ em instância central. É importante destacar que as salas de vacinas das Unidades de Saúde (US) se constituem em instâncias locais, cuja responsabilidade técnica e as ações relacionadas, no sentido de manter a qualidade da conservação e da administração dos imunobiológicos, são exclusivas da equipe de enfermagem ${ }^{4}$.

Um dos problemas básicos do dia a dia dos profissionais que atuam na sala de vacinação é a perda de imunobiológicos, a qual pode ser classificada como técnica ou física. Neste estudo, enfatizamos as perdas físicas, consideradas evitáveis, por ocorrerem quando há o descumprimento das normas e dos procedimentos adequados, como, por exemplo: quebra de frasco; procedimento inadequado (porta do refrigerador esquecida aberta, frascos de vacinas esquecidos na bancada, caixa térmica com vacinas mantidas de um dia para o outro, refrigerador sem termômetro para controle de temperatura etc.); falha no equipamento de refrigeração; falta de energia elétrica; validade vencida; falha no transporte; vandalismo etc ${ }^{5}$.

As perdas físicas podem e devem ser reduzidas com capacitação frequente dos profissionais que atuam na $\mathrm{RF}$, manutenção preventiva e corretiva dos equipamentos de refrigeração, controle de lotes e validade das vacinas, entre outras ações 5 .

No Paraná, a utilização do Sistema de Informação de Apuração de Imunobiológicos Utilizados (SI-AIU) permite verificar imunobiológicos utilizados, a movimentação de estoque e as perdas (técnicas e físicas) em todas as instâncias. Em 2013, uma análise do SI-AIU identificou que foram desprezadas, por causas evitáveis, mais de 700 mil doses de imunobiológicos. Tal fato pode ocasionar o desabastecimento desses produtos e, consequentemente, acarretar baixas coberturas vacinais e um possível aumento de casos de doenças imunopreveníveis, com prejuízo direto à população ${ }^{2}$.

Tal realidade nos remeteu à seguinte questão norteadora: como é o enfrentamento dos profissionais de enfermagem da instância local da RF de um município da $2^{\text {a }}$ Regional de Saúde - Metropolitana ( $\left.2^{\mathrm{a}} \mathrm{RS}\right)$ - frente às perdas evitáveis de imunobiológicos?

Os objetivos da pesquisa foram: identificar o município da $2^{\text {a }} \mathrm{RS}$ - Metropolitana do Paraná - com a maior ocorrência de perdas evitáveis de imunobiológicos notificada no período de 2011 a 2015; conhecer o processo de trabalho da equipe de enfermagem nas atividades relacionadas à RF de imunobiológicos na instância local do município com maior ocorrência de perdas evitáveis; e descrever as estratégias de enfrentamento às 
perdas evitáveis de imunobiológicos realizadas pelos profissionais de enfermagem nas instâncias locais da RF no cenário estudado.

\section{Métodos}

Foi realizado um estudo de caso do tipo exploratório documental ( $1^{\text {a }}$ fase) com abordagem quantitativa, e de campo ( $2^{\mathrm{a}}$ fase), com abordagem qualitativa. A primeira etapa configurou-se no reconhecimento do cenário a ser investigado, e a segunda num aprofundamento das singularidades e particularidades do cenário configurado como campo do estudo.

A pesquisa teve como base territorial a $2^{\text {a }}$ Regional de Saúde - Metropolitana do estado do Paraná -, constituída por 29 municípios. É a oitava região metropolitana mais populosa do Brasil, com 3.223.836 habitantes, e concentra $30,86 \%$ da população do estado, apresentando a maior movimentação e aplicação de imunobiológicos ${ }^{6}$.

$\mathrm{Na} 1^{\text {a }}$ fase, a pesquisa foi realizada utilizando-se dados secundários do SI-AIU e do Sistema de Informação do Programa Nacional de Imunização (SI-PNI), relativos ao período de 2011 a 2015. Esses sistemas permitem o controle da movimentação de imunobiológicos a partir da sala de vacina, apurando a utilização, as perdas técnicas e as perdas físicas (consideradas evitáveis), com consolidação dos dados nas instâncias municipal, estadual e nacional, bem como o controle de distribuição e estoque ${ }^{7}$.

Os municípios escolhidos para este levantamento foram os seis maiores da Região Metropolitana de Curitiba (RMC), com mais de 100.000 (cem mil) habitantes. Entre esses seis municípios foi identificado o que mais apresentou ocorrências informadas nos SI-AIU e SI-PNI, que levaram a perdas evitáveis no período analisado.

Uma vez reconhecido o município, a $2^{\mathrm{a}}$ fase foi realizada por meio de entrevistas coletivas/individuais em 21 das 23 US do município visitado, ocorridas entre maio e julho de 2016 e agendadas por telefone de acordo com a disponibilidade das equipes de enfermagem, em ambos os turnos.

$\mathrm{O}$ instrumento com o roteiro utilizado nas entrevistas foi dividido em duas partes: a primeira, com caracterização do profissional, tempo de formação e de atuação no serviço e se havia passado por capacitação em Sala de Vacinação em algum momento de sua atuação profissional; na segunda parte, buscamos conhecer, por meio de entrevistas, o processo de trabalho da equipe de enfermagem na RF local (sala de vacina), com ênfase em perdas vacinais: última ocorrência que levou à perda de imunobiológicos, procedimento adotado e qual a estratégia adequada para o enfrentamento de perdas evitáveis de imunobiológicos.

O estudo foi submetido à avaliação do Comitê de Ética em Pesquisa em Seres Humanos do Hospital do Trabalhador Secretaria de Estado da Saúde (Sesa), credenciado junto à Comissão Nacional de Ética em Pesquisa (Conep) do Ministério da Saúde, aprovado em 30 de julho de 2015, por meio do Parecer Consubstanciado de número 1.180.966. Foram respeitadas todas as normativas contidas na Resolução $\mathrm{n}^{\circ}$ $466 / 20128$.

Os critérios de inclusão na $2^{\mathrm{a}}$ fase foram: enfermeiros, técnicos e auxiliares de enfermagem de ambos os sexos, em atividade nas US e que desenvolvessem atividades gerenciais e/ou assistenciais na sala de vacinação das US, identificados por letras e números, de acordo com a categoria profissional.

Para análise dos dados da $1^{\mathrm{a}}$ etapa (abordagem quantitativa), foram utilizadas planilhas do programa Microsoft Excel $^{\circledR}$ contendo as variáveis: ano da ocorrência, local da ocorrência, imunobiológico, tipo de evento/perda, sendo, posteriormente, analisados por meio de estatística descritiva. $\mathrm{Na}$ $2^{\text {a }}$ etapa (abordagem qualitativa), os dados foram analisados por meio de análise de discurso, proposta por Fiorin 9 . 


\section{Resultados}

Com relação à $1^{\mathrm{a}}$ fase, após o levantamento nos bancos de dados, foram utilizados, para fins de análise, somente os números absolutos das perdas evitáveis de imunobiológicos, sendo então identificado o cenário da segunda fase: o município com o maior número de doses de imunobiológicos desprezados por perdas físicas (104.905 doses). No cenário estudado, tais perdas representaram $8,56 \%$ do total de doses recebidas (1.225.425 doses). As perdas evitáveis reconhecidas foram: quebra de frascos (6.655); falha de energia elétrica (4.072); falha no equipamento de refrigeração (5.567); validade vencida (87.035); procedimento inadequado/perdas em frascos abertos (688); falha no transporte (0); e outros motivos (888).

Quando analisados os motivos que levaram ao descarte de vacinas por causas evitáveis, observa-se que a perda por validade vencida representa $83 \%$ (87.035) do total de vacinas desprezadas. Em sete tipos de imunobiológicos, predominaram as seguintes ocorrências (quadro 1):

Quadro 1 - Identificação dos sete imunobiológicos desprezados por perdas evitáveis segundo causa - Quebra de Frasco (QF); Falha no Equipamento de refrigeração (FE); Procedimento Inadequado (PI); falha de Energia Elétrica (EE). Paraná, PR, Brasil, 2011 a 2015

\begin{tabular}{lllll}
\hline IMUNOBIOLÓGICO & QF & FE & PI & EE \\
\hline Soro antirrábico & $42,90 \%$ & $28,60 \%$ & & \\
DTP+Hib (tetravalente) & & $47,40 \%$ & $36,80 \%$ & \\
Raiva celular vero & $43,60 \%$ & $33,20 \%$ & & \\
Meningocócica conjugada C & & $43,00 \%$ & $35,60 \%$ \\
DTP+HB+Hib (pentavalente) & & $28,40 \%$ & $46,80 \%$ \\
Pneumocócica conjugada 10v. & & $32,20 \%$ & $30,80 \%$ \\
Poliomielite inativada & $17,15 \%$ & & \\
\hline
\end{tabular}

Para os outros 22 imunobiológicos validade vencida (gráfico 1). (53,7\%), a ocorrência predominante foi

Gráfico 1 - Proporção de perdas de imunobiológicos segundo ocorrência 'validade vencida'. PR., Brasil, 2016

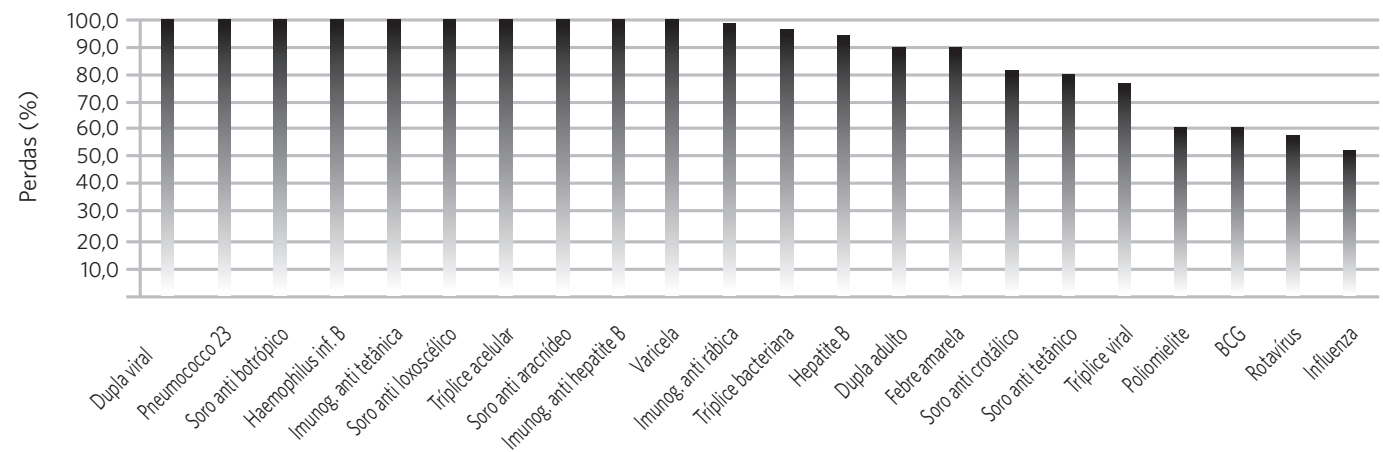


$\mathrm{Na}$ segunda fase, foram realizadas as entrevistas coletivas/individuais em 91\% (21) das US no município 'caso'. Duas US não foram visitadas, uma por recomendação da própria Secretaria de Saúde, por se tratar de área de risco, e outra por ser localizada em área rural de difícil acesso, com pouca atividade de vacinação e apenas um técnico de enfermagem.

De acordo com a coordenação das equipes de enfermagem municipal, os profissionais de enfermagem somam um total de 119 profissionais. Destes, 52 são enfermeiros, 60 são técnicos de enfermagem e sete são auxiliares de enfermagem.

O total de participantes foi de 54 profissionais de enfermagem (entre os que estavam nas US no horário agendado), sendo que foram realizadas dez entrevistas coletivas, com três a quatro participantes por entrevista, e 15 entrevistas individuais, com uma duração média de dez minutos, permitindo a participação de todos da equipe, sem prejudicar o atendimento da unidade (gráfico 2).

Gráfico 2 - Distribuição dos participantes entrevistados por categoria profissional. PR, Brasil, 2016

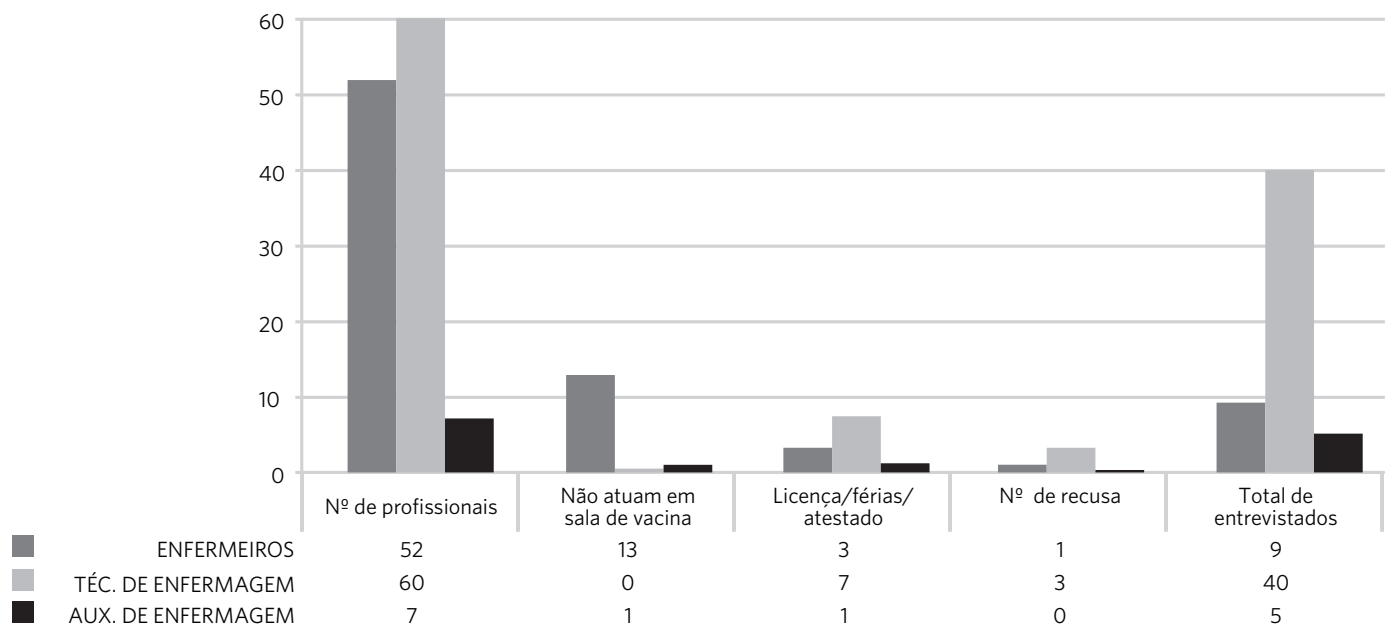

Dos enfermeiros, os nove entrevistados eram do sexo feminino. Destes, três estavam na faixa etária de 40 a 59 anos de idade e, na mesma proporção, apresentavam 21 anos ou mais de tempo de formação. Com relação ao tempo de sala de vacina, cinco dos enfermeiros atuavam entre 0 e 5 anos, e apenas dois relataram não terem sido capacitados em sala de vacinação.

Dos técnicos de enfermagem, foram entrevistados 40 profissionais, destes, somente dois eram do sexo masculino (5\%). $42 \%$ (17) estavam na faixa etária de 40 a 49 anos de idade, sendo que $30 \%$ (12) deles apresentaram tempo de formação de 11 a 15 anos. Quanto à atuação em sala de vacina, $42 \%$ (17) apresentavam de 0 a 5 anos, e $67 \%$ (27) profissionais foram capacitados há menos de um ano.

Dos cinco auxiliares de enfermagem entrevistados, apenas um (20\%) era do sexo masculino, $80 \%$ (4) estavam na faixa etária de 40 a 59 anos de idade, e $80 \%$ (4) com 21 anos ou mais de formação. Com relação ao tempo de atuação em sala de vacina, $40 \%$ (2) 
dos auxiliares de enfermagem atuavam entre 11 a 15 anos, e $40 \%$ (2) foram capacitados em sala de vacinação entre 4 a 5 anos.

Das 21 unidades visitadas, 15 (71,4\%) possuíam refrigerador específico para guarda de imunobiológicos em sua RF. As seis $(28,6 \%)$ restantes trabalhavam com o modelo de refrigerador de uso doméstico.

Após a identificação das ocorrências que levaram a perdas evitáveis de imunobiológicos e a caracterização dos profissionais entrevistados, foi realizada a leitura exaustiva das transcrições. Com relação à análise dos discursos, foram identificadas duas categorias: Processo de Trabalho (com duas figuras: organização da sala de vacina e aplicação de vacinas); e Perdas Evitáveis de Vacinas (com seis figuras: quebra de frasco, falta de energia elétrica, procedimento inadequado, falha no equipamento de refrigeração, depredação de patrimônio/outros e expiração do prazo de validade/validade vencida).

Abaixo, apresentamos as categorias, algumas frases temáticas $\mathrm{e}$ as figuras encontradas.

\section{Categoria processo de trabalho}

Figura: Organização da sala de vacinas

Hoje, temos um aparelho de refrigerador que dispensa o uso de colocar o gelo para temporizar e depois pôr a vacina dentro do isopor. [...]. (Téc. 10).

Quando chego, tiro a temperatura e o gelox para degelar, espero uns minutos para ele entrar na temperatura [...] começo pôr a vacina e vai até às 16 horas. [...]. (Téc. 06).

Figura: Aplicação de vacinas

[...] vai fazer a aplicação, você faz a lavagem das mãos, vai lá dentro da geladeira e pega a vacina [...]. (Téc. 05).

[...] chegou uma criança para tomar a vacina, a gente pega, vai lá, retira o frasquinho, aspira a dose e guarda [...]. (Téc. 09).

\section{Categoria perdas evitáveis de vacinas}

Figura: quebra de frasco

Já quebrei um frasquinho de vacina [...]. (Aux. 01).

Às vezes, acontece quebra de um frasco, mas é muito pouco [...]. (Téc. 17).

Figura: falta de energia elétrica

[...] falta de energia, a gente teve perda de um lote muito grande $[. .$.$] , em torno de 300$ doses. (Téc. 07).

Só falta de energia que é comum no bairro. Quando chove, acontece muito de ficar sem luz [...]. (Téc. 39).

Figura: procedimento inadequado

Aconteceu uma vez esquecimento da porta da geladeira aberta [...]. (Téc. 03).

[...] esquecimento no final do ano de repor as vacinas da caixa térmica na geladeira [...]. (Téc. 37).

Figura: falha no equipamento de refrigeração

[...] a geladeira, quando não era essa, ou esquentava demais ou gelava demais, e a gente perdia vacina [...]. (Téc. 09).

Figura: depredação do patrimônio público

[...] vândalos entraram, além de mexer em tudo, roubar nossas coisas, deixaram a geladeira aberta. (Téc. 05).

Já teve vandalismo, desligaram a luz [...]. (Téc. 03). 
Figura: expiração do prazo de validade

[...] dentre todos estes, a validade do laboratório é o que mais acontece. A vacina vem para a gente muito próximo de vencer [...]. (Téc. 04).

[...] prazo de validade, porque chegou um lote próximo de vencer, e não conseguimos utilizar tudo [...]. (Téc. 36).

Quanto à primeira figura, 'Organização da sala de vacinas' (categoria Processo de Trabalho), foram diferenciados os discursos entre os profissionais que utilizavam diferentes tipos de refrigeradores, uma vez que os profissionais que trabalham com o refrigerador do tipo doméstico relatam o tempo despendido entre o preparo da caixa térmica e o início das atividades de vacinação.

Para a segunda figura encontrada, 'Aplicação de vacinas', os discursos também mostram essa praticidade no processo de trabalho quando utilizados os refrigeradores específicos para o armazenamento de imunobiológicos.

Com relação à segunda categoria, 'Perdas evitáveis de vacinas', apresentamos, abaixo, as ocorrências (gráfico 3) relativas à pergunta: quais perdas evitáveis de vacina você já vivenciou durante seu trabalho na unidade de saúde?

Gráfico 3 - Tipos de perdas evitáveis de imunobiológicos vivenciadas em números absolutos. PR, Brasil, 2016

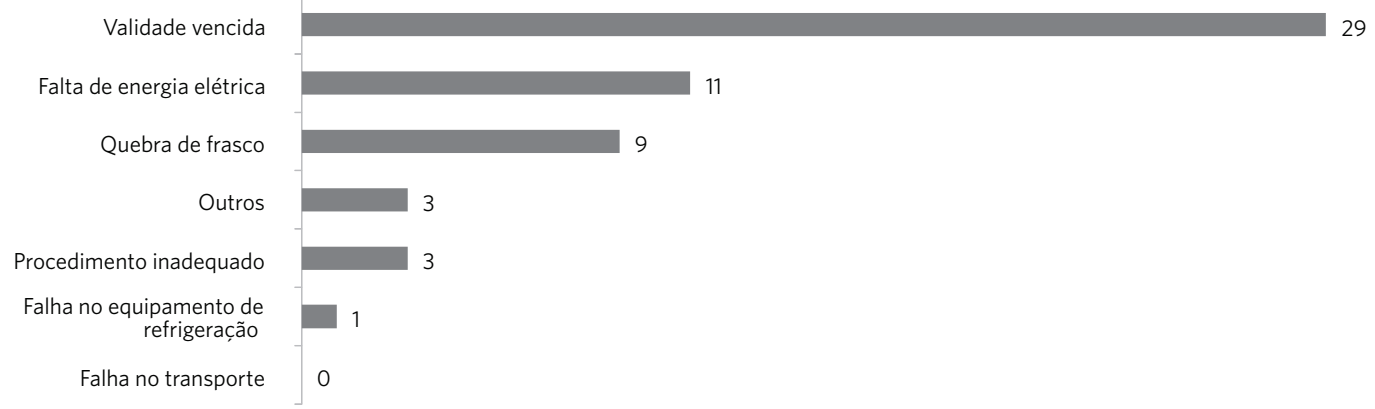

Pode-se observar que a centralidade dos discursos dos entrevistados está na vivência de perdas por validade vencida, a qual representou $52 \%$ do total de perdas evitáveis. Tal informação vai ao encontro dos dados levantados nos sistemas de informação, nos quais $83 \%$ do total das vacinas desprezadas foi por validade vencida. Os três registros de perda por procedimento inadequado ocorreram pelos seguintes motivos: caixa térmica esquecida fora do refrigerador no final do expediente; frasco da vacina BCG deixado aberto derramando nas demais vacinas; porta do refrigerador esquecida aberta. Os três registros de perda por outros motivos ocorreram: dois por vandalismo e a outra porque uma criança tirou o cabo de energia do refrigerador da tomada.

\section{Discussão}

O enfermeiro é responsável pela supervisão e pelo monitoramento do trabalho desenvolvido na RF local, bem como pelo processo de educação permanente, tendo 
responsabilidade técnico-administrativa pelas atividades realizadas na sala de vacinação, o que inclui o desenvolvimento de habilidades e competências da equipe. Os vacinadores, na maioria, técnicos ou auxiliares de enfermagem, são imprescindíveis ao processo, sendo que a quantidade de vacinadores varia de acordo com o porte do serviço e com o tamanho da população a ser assistida ${ }^{10}$.

Nos resultados encontrados, percebeu-se que a quantidade de profissionais e a capacitação técnica não estavam em consonância com normativas do PNI, uma vez que nem todos os vacinadores passaram por capacitação em sala de vacinas.

Estudos brasileiros avaliaram as condições de acondicionamento e refrigeração dos imunobiológicos, descrevendo a ocorrência do descumprimento de recomendações, sendo que falhas nesse processo foram significativas nas US, colocando em risco a eficácia da imunização. Entre estes estudos, destacamos dois: no Nordeste brasileiro, foram encontrados registros de temperatura dos refrigeradores das salas de vacina fora do preconizado"1, e em Campinas (SP), foram observadas inconformidades na organização interna dos refrigeradores, além de distorção da descrição técnica a respeito do degelo desses equipamentos ${ }^{12}$.

Tais estudos não corroboram a realidade do cenário investigado, no qual, das ocorrências de perdas evitáveis de imunobiológicos identificadas, a que se destacou foi a perda por validade vencida, com mais de $87 \mathrm{mil}$ doses desprezadas, dado confirmado nos discursos dos profissionais.

Segundo Aranda e Moraes ${ }^{13}$, conhecer como se desenvolve a prática cotidiana de enfrentamento das perdas evitáveis de imunobiológicos é imprescindível para análise do processo de trabalho nesse domínio, bem como para subsidiar os gestores da RF quanto à capacitação de pessoal em sala de vacinação, elaboração de manuais, exercícios práticos e abordagens técnicas sobre o calendário vacinal e a melhoria do serviço prestado.
Pode-se observar que a maioria dos vacinadores (67\%) passou por capacitação técnica em sala de vacinas a menos de um ano, ação fundamental para se atuar na sala de vacinas, uma vez que, durante a formação profissional tanto de técnicos como de enfermeiros, não se aborda a imunização em todas as peculiaridades que ela exige. Embora os profissionais de enfermagem tenham formação curricular e amparo legal para exercer essa atividade, ao atuarem em imunização, devem aprofundar seus conhecimentos nas bases do PNI, adquirir boas práticas para administração e conservação de imunobiológicos, respeitando as recomendações pertinentes a cada uma delas ${ }^{\mathbf{1 4}}$.

Outro ponto a ser discutido é a rotatividade dos profissionais. Nem sempre o profissional capacitado é aquele que está atuando na sala de vacinas. Por esse motivo, faz-se necessária a capacitação em imunização de todos os profissionais do serviço.

O programa de imunização é fundamentalmente exercido por profissionais de enfermagem na maioria dos municípios do Brasil, e a supervisão das atividades deve ser exercida sistematicamente pelo enfermeiro, incluindo os aspectos organizacionais e a capacitação profissional14.

Pode-se observar que 25\% (13) dos enfermeiros não atuam na sala de vacinação do município, mesmo sendo os responsáveis pelas ações nela executadas. Muitos até relatam a necessidade de estarem mais presentes, porém, a demanda excessiva e a falta de profissionais fazem com que diminuam a participação nas atividades relacionadas à sala de vacinação.

O perfil dos participantes é de profissionais com mais de 20 anos de formação e com faixa etária predominante de 40 anos ou mais. Esses dados vão ao encontro dos levantados em um estudo que mostra que a maioria das ocorrências de erros em procedimentos de imunização acontece com profissionais que apresentam uma média de idade de 36,5 anos, com tempo de formação 
entre um e dez anos ${ }^{\mathbf{1 3}}$. Outro estudo, realizado no interior do estado de São Paulo, mostra uma realidade diferente: erros de medicação ocorrem com maior propensão entre profissionais experientes, pois se sentem convencidos de que seu julgamento e sua decisão são os mais corretos ${ }^{15}$.

No entanto, podemos observar que, no município estudado, o processo de trabalho da equipe de enfermagem nas salas de vacinas não está diretamente relacionado com o número de perdas evitáveis de imunobiológicos por validade vencida. Essa situação não está sob responsabilidade da instância local, uma vez que o lote de vacinas é recebido com curto prazo de validade, e o procedimento de distribuição, assim como o processo de aquisição de imunobiológicos, está sob a responsabilidade da instância estadual.

Vale destacar que as duas US não visitadas não afetam o resultado da pesquisa, uma vez que, conforme a Coordenação Geral das equipes de enfermagem, uma Unidade de Saúde é de área rural, com apenas uma técnica de enfermagem para o atendimento, havendo baixa movimentação de imunobiológico. Já a outra Unidade não foi recomendada para a visita por situar-se em área de risco, em virtude da presença constante de grupos relacionados ao tráfico de drogas e da consequente violência entre gangues rivais, onde o procedimento de vacinação é realizado por dois técnicos de enfermagem, e o movimento de imunobiológicos é baixo pelas próprias características da comunidade abrangente.

Algumas estratégias de enfrentamento para evitar a perda de vacinas por validade vencida foram propostas pelos profissionais, com destaque para o melhor gerenciamento de lotes na instância superior, o que possibilitaria a entrega das vacinas com maior prazo de validade para utilização. Tal perspectiva se faz primordial, uma vez que $46 \%$ dos participantes relataram vivenciar o descarte de vacinas por validade vencida.

Como podemos observar nos discursos, foram vivenciados praticamente todos os tipos de perdas evitáveis de vacinas, com exceção das causadas por falha no transporte das vacinas. $\mathrm{O}$ tipo de perda menos vivenciada foi o procedimento inadequado, o que nos faz refletir se este tipo de perda realmente ocorre pouco ou se a equipe tem dificuldade em identificar a sua ocorrência.

A literatura registra uma ampla variedade de estudos a respeito de armazenamento, conservação, manipulação de vacinas, prática em RF, porém, pouco se conhece sobre perdas vacinais. Em geral, quando esse tema é abordado, há ênfase nas razões externas relacionadas aos sistemas de informação e ao PNI, e não ao processo de trabalho na sala de vacina ${ }^{16}$.

Um estudo realizado no Canadá demonstrou que o custo estimado de perdas de vacinas foi de três milhões de dólares ao ano, e que $20 \%$ dos serviços de saúde não cumprem as condições mínimas de armazenamento e manuseio das vacinas, sendo que cerca de $4 \%$ das vacinas são desprezadas por vencimento do prazo de validade ${ }^{16}$.

Destarte, foi possível perceber que as estratégias de enfrentamento das perdas vacinais se localizam para além das salas de vacina, o que nos leva a pensar em outras investigações necessárias para tal enfrentamento.

\section{Conclusões}

O planejamento no uso de recursos públicos para aquisição, distribuição e aplicação dos imunobiológicos impacta diretamente a eficácia da ação do PNI. O uso dos insumos desse programa, sem avaliação, em todo o processo pode levar ao desabastecimento de produtos essenciais ou à perda de produtos pela incapacidade de consumi-los nos prazos de validade, levando ao aumento de perdas por vários motivos.

O conhecimento adquirido acerca do processo de trabalho da equipe de enfermagem no cenário estudado poderá subsidiar novas 
ações no gerenciamento dos imunobiológicos, que tanto minimizem perdas evitáveis de vacinas como possibilitem enfrentamento adequado das doenças imunopreveníveis.

Perdas vacinais são esperadas. A questão fundamental consiste no fato dessas perdas serem ou não evitáveis, para as quais cabe a adoção de medidas de prevenção. A perda de vacinas em frascos ainda fechados fica atribuída a problemas de gestão de estoque, o que pode ser rapidamente minimizado. Já as perdas em frascos abertos não podem ser eliminadas, porém, a adoção de políticas e práticas melhoradas em imunização podem reduzi-las substancialmente ${ }^{15}$.

São necessários novos estudos nesse domínio, uma vez há escassez de literatura fora das publicações oficiais acerca de perdas evitáveis vacinais.

A análise dos discursos trouxe vantagens: dar voz a um grupo por meio da identificação das figuras que apareceram nas falas analisadas. Faz-se necessário ressaltar que as questões político-ideológicas afetas ao processo de trabalho (compra, estoque e distribuição) em sala de vacina foram pouco evidenciadas nos discursos emitidos pelos atores sociais envolvidos no estudo.

Tal realidade indica uma necessidade de desenvolver junto à equipe de enfermagem os outros componentes de processo de trabalho: administrar, ensinar, pesquisar e participar politicamente, sendo este último um elemento fundamental do trabalho, uma vez que a enfermagem precisa se instrumentalizar para minimizar as perdas vacinais por validade vencida.

Com relação às possíveis limitações do estudo, destacamos as prováveis subnotificações nos sistemas envolvidos nos registros de perdas vacinais, bem como uma baixa adesão às entrevistas. 


\section{Referências}

1. Brasil. Ministério da Saúde. Uma análise da situação de saúde e dos 40 anos do Programa Nacional de Imunizações. Brasília, DF: Ministério da Saúde; 2013.

2. Oliveira VC, Caveião C, Crosewski F. Gerenciamento de enfermagem no controle de perdas evitáveis de imunobiológicos. Cogitare Enferm. 2014; 9(4):679-686

3. Ponte CF. Vacinação, controle de qualidade e produção de vacinas no Brasil a partir de 1960. Hist. Cienc.Saúde. 2003; 10(supl.2):619-653.

4. Brasil. Ministério da Saúde. Secretaria de Vigilância em Saúde. Departamento de Vigilância de Doenças Transmissíveis. Coordenação Geral do Programa Nacional de Imunizações. Manual de Rede de Frio. Brasília, DF: Ministério da Saúde; 2011.

5. Pereira DDS, Neves EB, Gemelli M, et al. Análise da taxa de utilização e perda de vacinas no programa nacional de imunização. Cad. Saúde Colet. 2013; 21(4):420-424.

6. Coordenação da Região Metropolitana de Curitiba [internet]. Região Metropolitana de Curitiba [acesso em 2015 set 15]. Curitiba: Comec; [data desconhecida]. Disponível em: http://www.comec.pr.gov.br/ modules/conteudo/conteudo.php?conteudo=123.

7. Brasil. Ministério da Saúde. Secretaria de Vigilância em Saúde. Departamento de Vigilância Epidemiológica. Sistema de Informação de Apuração de Imunobiológicos Utilizados - SIAIU. Manual do Usuário. Brasília, DF: Ministério da Saúde, 2007.

8. Brasil. Ministério da Saúde. Resolução n. 466, de 12 de dezembro de 2012. Diretrizes e normas regulamentadoras de pesquisa envolvendo seres humanos. Diário Oficial da União. 12 Dez 2012.
9. Fiorin JL. Linguagem e ideologia. 8. ed. São Paulo: Ática; 2007.

10. Oliveira VC, Guimarães EAA, Cavalcante RB, et al. Conservação de vacina em unidades públicas de saúde: uma revisão integrativa. Rev. Enf. Ref. 2013; (9):45-54.

11. Luna GLM, Vieira LJES, Sousa PF, et al. Aspectos relacionados à administração e conservação de vacinas em centros de saúde no Nordeste do Brasil. Ciênc Saúde Colet. 2011; 16(2):513-552.

12. Ribeiro DO, Castro F, Ferreira GC, et al. Qualidade na conservação e armazenamento dos imunobiológicos da rede básica do Distrito Sul de Campinas. J Health Sci Inst, 2010; 28(1):21-28.

13. Aranda CMSS, Moraes JC. Rede de frio para a conservação de vacinas em unidades públicas do município de São Paulo: conhecimento e prática. Rev. bras. Epidemiol. 2006; 9(2):172-185.

14. Rodrigues IC, Paschoalotto AA, Brunieira ELL. Procedimentos inadequados em sala de vacina: a realidade da região da São José de Rio Preto. Bol. Epidemiol. Paul. (Online). 2012; 9(100):16-28.

15. Silva BK, Silva JS, Gobbo AFF, et al. Erros de medicação: condutas e propostas de intervenção na perspectiva da equipe de enfermagem. Rev. Eletrôn. Enfer. [internet]. 2007 [acesso em 2016 fev 15]; 9(3):712-723. Disponível em: http://www.fen.ufg. br/revista/v9/n3/v9n3all.htm.

16. Samad SA. Perdas vacinais: razões e prevalências em quatro unidades federadas do Brasil [tese]. São Paulo: Universidade Federal de São Paulo; 2011.85 p.

Recebido em 25/10/2017

Aprovado em 03/02/2018

Conflito de interesses: inexistente

Suporte financeiro: não houve 